\title{
Relationship between Serum Bilirubin Levels and Metabolic Syndrome in Patients with Schizophrenia Spectrum Disorders
}

\author{
Filiz Karadag ${ }^{1}$, Ceyhan Balci Sengul $\left.\right|^{2}$, Yasar Enli ${ }^{3}$, Kamuran Karakulah ${ }^{4}$, Huseyin Alacam², Bunyamin Kaptanoglu ${ }^{5}$, \\ Ozgur Kalkanci ${ }^{6}$, Hasan Herken ${ }^{2}$ \\ ${ }^{1}$ Department of Psychiatry, Gazi University Medical Faculty, Ankara, Departments of ${ }^{2}$ Psychiatry and ${ }^{3}$ Biochemistry, Pamukkale University Medical \\ Faculty, Denizli, ${ }^{4}$ Department of Psychiatry, Tokat Dr. Cevdet Aykan Mental Health Hospital, Tokat, ${ }^{5}$ Department of Biochemistry, Fatih University \\ Medical Faculty, Istanbul, ${ }^{6}$ Department of Psychiatry, Denizli State Hospital, Denizli, Turkey
}

\begin{abstract}
Objective: We investigated the relationship between serum bilirubin levels and metabolic syndrome (MetS), and the longitudinal effects of baseline serum bilirubin concentrations on MetS in patients with schizophrenia spectrum disorders undergoing atypical antipsychotics.

Methods: The sample of this study consisted of 131 patients with schizophrenia spectrum disorders. Waist circumference, blood pressure, and levels of triglycerides, high-density lipoprotein cholesterol, fasting glucose, and insulin were evaluated at baseline and at month six. Serum bilirubin levels were measured at baseline. Serum bilirubin levels of the patients with and without MetS criteria were compared. We also compared patients with high and low bilirubin levels (upper and lower 50th percentiles of serum bilirubin levels) in terms of MetS criteria, MetS frequency, and course of MetS.

Results: Serum direct bilirubin levels were more consistently related to MetS and MetS-related variables. The waist circumference and triglyceride criteria for MetS were significantly related to low serum direct bilirubin at baseline; waist circumference and fasting glucose criteria, and insulin resistance were associated with low serum direct bilirubin at follow-up. MetS diagnosis and the presence of the waist circumference criterion were more frequent at the baseline and the follow-up in low bilirubin group. At the end of the follow-up period, the rate of reverse MetS was significantly higher in the high bilirubin group.

Conclusion: Our results have suggested that serum direct bilirubin levels showed a more reliable and stable relationship with abdominal obesity for MetS components. in patients with schizophrenia spectrum disorders using antipsychotics. Further studies are required.
\end{abstract}

KEY WORDS: Schizophrenia; Metabolic syndrome; Bilirubin; Atypical antipsychotics.

\section{INTRODUCTION}

Metabolic syndrome (MetS) is a combination of metabolic dysfunctions including abdominal or visceral adiposity, hypertension, glucose, and lipid abnormalities that increase the risk for cardiovascular disease and diabetes. ${ }^{1)}$ MetS is a growing concern for routine psychiatric care when treating patients with schizophrenia because these metabolic abnormalities are regarded as a major risk factor for cardiovascular diseases and mortality. ${ }^{2-4)}$ Factors predisposing people with schizophrenia to MetS are complicated. Particularly second generation anti-

\footnotetext{
Received: April 6, 2016/Revised: June 16, 2016

Accepted: June 22, 2016

Address for correspondence: Huseyin Alacam, MD

Department of Psychiatry, Pamukkale University Medical Faculty, Kınıklı, Denizli 20070, Turkey

Tel: +90-258-296-60-00, Fax: +90-258-296-60-01

E-mail: dr.huseyinalacam@hotmail.com
}

psychotics have been shown to greatly influence metabolic risk factors leading to weight gain and impairing glucose and lipid metabolism. ${ }^{5)}$ The other risk factors among patients with schizophrenia are attributed to poor dietary habits, unhealthy lifestyle and physical inactivity, possibly related to negative symptoms of schizophrenia. ${ }^{2}$ Weight gain and metabolic side effects of atypical antipsychotics also leads to non-adherence or rejection of treatment by the schizophrenia patients. ${ }^{6}$ )

Bilirubin, the end product of heme metabolism, is an endogenous antioxidant with anti-inflammatory properties. High serum bilirubin concentrations are associated with increased total antioxidant capacity and offer protection against oxidative stress-induced diseases. ${ }^{7}$ MetS is characterized by enhanced low-grade systemic inflammation and oxidative stress. During the past few years, studies have shown that serum bilirubin levels are

() This is an Open-Access article distributed under the terms of the Creative Commons Attribution Non-Commercial License (http://creativecommons.org/licenses/by-nc/4.0) which permits unrestricted non-commercial use, distribution, and reproduction in any medium, provided the original work is properly cited. 
inversely associated with MetS and systemic inflammation in adults, ${ }^{8-11)}$ children and adolescents. ${ }^{11)}$ It have been reported that especially, abdominal obesity has been correlated with low serum bilirubin levels. ${ }^{11-13)}$ Low serum bilirubin has been proven to be associated with increased carotid intima media thickness ${ }^{8,14)}$ and peripheral arterial disease. ${ }^{15-17)}$ In one study, low prevalence of ischemic heart disease in patients with Gilbert syndrome, a genetic disorder causing mild to moderate elevations of unconjugated bilirubin, was detected. ${ }^{18)}$ Bilirubin serum levels are determined by genetic factors (intrinsic activity of enzymatic steps in bilirubin homeostasis), serum albumin concentration and its bilirubin-binding properties, and external factors such as dietary status, fasting, tobacco smoking, intake of drugs or plant products, living at altitude, age, fitness level, and general health status. ${ }^{8)}$ However, it has been reported that antipsychotic drugs do not cause significant changes in serum bilirubin levels. ${ }^{19)}$

To our knowledge, the relationship between the bilirubin levels and MetS has not been studied in patients with schizophrenia or schizoaffective disorder using antipsychotics. In this naturalistic follow-up study, the relationship between the bilirubin levels and MetS-associated parameters, such as the frequency and course of MetS, was investigated in patients with schizophrenia spectrum disorders using antipsychotics. We also planned to investigate, if there is a relationship as we expected, the longitudinal effects of baseline serum bilirubin concentrations on incident MetS during six-month follow-up period. We hope that our results will provide preliminary information on whether or not bilirubin levels are a potential predictor for MetS in patients with schizophrenia spectrum disorders using antipsychotics. In the follow-up period, we examined whether serum bilirubin levels were associated with the development or reversal of MetS in these patients.

\section{METHODS}

\section{Study Design and Population}

The sample of the present study consisted of 151 patients who were diagnosed with schizophrenia or schizoaffective disorder according to the Diagnostic and Statistical Manual of Mental Disorders 4th edition (DSM-IV) criteria, aged 18-65 years, and receiving atypical antipsychotics for at least 12 weeks. The study conducted on a subgroup of patients who enrolled in another prospective naturalistic study investigating the association between antipsychotic medication use and MetS in patients with schizophrenia spectrum disorders. ${ }^{20)}$ This study supported by the
Committee of Scientific Research Projects of Pamukkale University (Project Number: 2008TPF029). The patients were recruited from two treatment settings (Pamukkale University, Psychotic Disorder Outpatient Clinics and Denizli State Hospital, Turkey). Patients with the following exclusion criteria were not recruited: psychotic disorders or mood disorders due to a general medical condition, dementia, substance abuse, or known hepatic dysfunction according to medical records.

The patients were evaluated at baseline and at month six. Antipsychotic medications and the severity of positive and negative symptoms were also recorded. ${ }^{21,22)}$ At baseline visit, all patients received a full clinical examination, metabolic screening for MetS parameters and measurement of serum bilirubin levels. Following the exclusion of 8 patients with serum direct bilirubin values above the normal range $(0.1-0.3 \mathrm{mg} / \mathrm{dl})$ and 12 patients with indirect bilirubin levels above the normal range $(0.2-0.7 \mathrm{mg} / \mathrm{dl})$, the baseline sample included 131 patients (78 male, 53 female) and the follow-up sample included 121 patients (72 male, 49 female).

The Medical Ethics Committee of Pamukkale University approved the study protocol (protocol no: 4837, 21.10.2008). Objectives and procedures of this study were explained to all patients; patients were informed that their ongoing treatments would not be affected by study procedures and written informed consent were obtained from all subjects prior to participation of the study.

All patients informed about their baseline measurement, and relevant recommendations were given. For example, patients with triglyceride (TG) and glucose levels meeting the MetS criteria were informed of the associated cardiovascular risk, advised to seek an endocrinology referral, and recommended to avoid high-calorie foods, to lose weight, to exercise, and to quit smoking.

\section{Anthropometric Measurements}

Weight was measured with a spring balance that was kept on a firm horizontal surface. Subjects wore light clothing and stood upright without shoes, and weight was recorded to the nearest $0.5 \mathrm{~kg}$. Height was measured with a tape to the nearest $0.1 \mathrm{~cm}$. Subjects were requested to stand upright without shoes with their backs against the wall, heels together, and eyes directed forward. Waist circumference was measured in a standing position halfway between the costal edge and iliac crest. Body mass index (BMI) was calculated as the body weight in kilograms divided by the height in meters squared. ${ }^{23)}$ The blood pressure 
measurements were obtained from the right arm with the participant in the sitting position using a mercury sphygmomanometer after 15 minutes of rest.

\section{Serum Measurements}

Overnight fasting blood samples were drawn and analyzed. Serum was immediately separated and stored at $-20^{\circ} \mathrm{C}$ until subsequent analysis. Serum concentrations of direct (conjugated with glucuronic acid), indirect (unconjugated), and total bilirubin (includes both direct and indirect bilirubin), fasting glucose, TG, and high-density lipoprotein (HDL) cholesterol were measured on an AU 680 Chemistry System analyzer (Beckman Coulter, Nyon, Switzerland) using commercial kits. Serum insulin concentrations were analyzed on an ARCHITECT i2000SR analyzer (Abbott Diagnostics, Abbott Park, IL, USA) using commercial kits. The homeostasis model assessment (HOMA) was used as a measure of insulin resistance (HOMA-IR). HOMA-IR was calculated using the formula: HOMA-IR $=$ glucose $(\mathrm{mmol} / \mathrm{L}) \times$ insulin $(\mu \mathrm{U} / \mathrm{ml}) / 22.5]$ and insulin resistance was defined as HOMA-IR $>2.7 .{ }^{19)}$ All body and biochemical measurements were repeated at follow-up visit.

\section{Definition of Metabolic Syndrome}

MetS was defined according to ATP III A (Adult Treatment Panel III A) criteria, ${ }^{20)}$ which takes into account the following five components: Fasting triglycerides (FTG) $>150 \mathrm{mg} / \mathrm{dl}$; HDL $<40 \mathrm{mg} / \mathrm{dL}$ (men) or $<50$ $\mathrm{mg} / \mathrm{dl}$ (women); blood pressure (systolic blood pressure/diastolic blood pressure) $>130 / 85 \mathrm{mmHg}$ or on antihypertensive medication; fasting glucose $>100 \mathrm{mg} / \mathrm{dl}$ or on insulin or hypoglycemic medication; and waist circumference $>102 \mathrm{~cm}$ (men) or $>88 \mathrm{~cm}$ (women). Patients having three or more of the criteria were identified as having a MetS diagnosis.

\section{Statistical Analysis}

Initially, in order to explore which bilirubin components would be associated with MetS, the means of serum bilirubin levels (direct, indirect, and total) were compared between the patients with and without MetS criteria. As bilirubin values did not show a normal distribution, mean bilirubin levels of the groups were compared using the Mann-Whitney $U$ test.

These analyses revealed a consistent relationship between direct serum bilirubin levels and MetS criteria, therefore we performed subsequent analyses based on a recent report on the lower risk of MetS in patients with se- rum bilirubin levels at upper percentiles. ${ }^{10)}$ The patients within the lower 50th percentile of serum direct bilirubin levels (low bilirubin group) were compared to those within the upper 50th percentile (high bilirubin group) in terms of MetS criteria using the chi-square test.

The stability of the relationship between serum bilirubin levels and MetS was tested by examining the relationship between the baseline serum bilirubin levels and MetS associated parameters at follow-up.

The relationship between bilirubin levels and MetS criteria was also investigated using partial correlation analysis while controlling for the effect of age. $p<0.05$ was accepted to be statistically significant. Statistical analyses were performed using SPSS software 17.0 for Windows (SPSS Inc., Chicago, IL, USA).

\section{RESULTS}

Bilirubin levels were measured in 151 patients at the beginning of study. For baseline sample, average age, mean duration of disease, and mean number of hospitalizations were determined to be $38.98 \pm 12.21$ years, $13.32 \pm 9.74$ years, and $2.84 \pm 3.57$ respectively. Frequency of MetS diagnosis was $45.8 \%$ at the baseline and $46.6 \%$ at follow-up (Table 1). Frequency of MetS diagnosis was not significantly different between men and women in either visit. Criteria for MetS diagnosis were met by $47.2 \%$ of

Table 1. Sample characteristics

\begin{tabular}{lc}
\hline \multicolumn{1}{c}{ Characteristic } & Data \\
\hline Gender & \\
Female & $53(40.5)$ \\
Male & $78(59.5)$ \\
MetS diagnosis & \\
Baseline MetS present & $60(45.8)$ \\
Free of MetS & $71(54.2)$ \\
Follow-up MetS present & $61(46.6)$ \\
Free of MetS at follow-up & $60(45.8)$ \\
Course of MetS & $46(75.4)$ \\
Continued MetS at both visits & $15(24.6)$ \\
Newly developed MetS at follow-up visit & $51(85.0)$ \\
Free of MetS at both visits & $9(15.0)$ \\
Reverse MetS at follow-up visit & \\
Medications & $63(48.1)$ \\
Atypical antipsychotics & $36(27.5)$ \\
Atypical combinations & $32(24.4)$ \\
Atypical-typical combinations & $65(49.6)$ \\
Smoking & $66(50.4)$ \\
Yes &
\end{tabular}

Values are presented as number (\%). Mets, metabolic syndrome. 
women and $49.9 \%$ of men at baseline and $49.9 \%$ of women and $52.8 \%$ of men at follow-up $(p>0.05)$. At the baseline evaluation, average age of the patients with MetS (43.95 \pm 11.01 years) was significantly higher than those without MetS $(34.78 \pm 11.65$ years $)(p=0.000)$.

Most of the patients with MetS (75.4\%) at follow-up visit were patients who met criteria for MetS in both visits and $24.6 \%$ were patients who developed MetS within the six months between visits. Most of the patients (85\%) without MetS at follow-up visit were free of MetS at baseline and $15 \%$ were reverse MetS patients. Most of our patients were treated with single or combined atypical antipsychotics (Table 1).

\section{The Relationship between Serum Bilirubin Levels and MetS Diagnosis, and Its Stability}

The mean serum direct bilirubin and total bilirubin levels of the patients with MetS diagnosis were significantly lower than those free of MetS. This relationship was also evident at follow-up. Regarding the course of MetS, the patients who met the diagnosis of MetS in both visits had significantly lower direct bilirubin levels compared to patients with reversed MetS diagnosis at the end of the follow-up period (Table 2).

Serum direct bilirubin levels were also significantly lower in patients who met the MetS criterion for waist circumference at baseline as compared to patients who were free of this criterion. The presence of TG level criteria for

Table 2. The relationship between serum bilirubin levels and MetS diagnosis at baseline and follow-up

\begin{tabular}{|c|c|c|c|c|c|c|c|}
\hline Variable & Number & $\begin{array}{c}\text { Direct bilirubin } \\
\text { level }\end{array}$ & $p$ value & $\begin{array}{l}\text { Indirect bilirubin } \\
\text { level }\end{array}$ & $p$ value & $\begin{array}{l}\text { Total bilirubin } \\
\text { level }\end{array}$ & $p$ value \\
\hline \multicolumn{8}{|l|}{ Baseline } \\
\hline MetS present & 60 & $0.075 \pm 0.066$ & & $0.264 \pm 0.127$ & & $0.347 \pm 0.142$ & \\
\hline Free of MetS & 71 & $0.121 \pm 0.093$ & 0.002 & $0.290 \pm 0.178$ & 0.193 & $0.421 \pm 0.203$ & 0.011 \\
\hline \multicolumn{8}{|l|}{ Individual MetS criteria } \\
\hline BP criterion present & 35 & $0.105 \pm 0.081$ & & $0,267 \pm 0.128$ & & $0.369 \pm 0.166$ & \\
\hline Free of BP criterion & 96 & $0.103 \pm 0.087$ & 0.958 & $0.292 \pm 0.166$ & 0.339 & $0.397 \pm 0.198$ & 0.429 \\
\hline FG criterion present & 46 & $0.091 \pm 0.082$ & & $0.267 \pm 0.137$ & & $0.358 \pm 0.154$ & \\
\hline Free of FG criterion & 85 & $0.102 \pm 0.083$ & 0.341 & $0.291 \pm 0.165$ & 0.399 & $0.397 \pm 0.201$ & 0.249 \\
\hline WC criterion present & 80 & $0.085 \pm 0.075$ & & $0.248 \pm 0.141$ & & $0.333 \pm 0.161$ & \\
\hline Free of WC criterion & 51 & $0.126 \pm 0.090$ & 0.006 & $0.337 \pm 0.163$ & 0.001 & $0.464 \pm 0.198$ & 0.000 \\
\hline HDL criterion present & 96 & $0.095 \pm 0.082$ & & $0.281 \pm 0.144$ & & $0.377 \pm 0.178$ & \\
\hline Free of HDL criterion & 35 & $0.115 \pm 0.087$ & 0.193 & $0.287 \pm 0.186$ & 0.843 & $0.4034 \pm 0.212$ & 0.488 \\
\hline TG criterion present & 57 & $0.076 \pm 0.068$ & & $0.277 \pm 0.116$ & & $0.353 \pm 0.136$ & \\
\hline Free of TG criterion & 74 & $0.120 \pm 0.090$ & 0.003 & $0.287 \pm 0.181$ & 0.707 & $0.408 \pm 0.216$ & 0.081 \\
\hline Insulin resistance present & 33 & $0.087 \pm 0.083$ & & $0.276 \pm 0.151$ & & $0.363 \pm 0.165$ & \\
\hline No insulin resistance & 98 & $0.105 \pm 0.084$ & 0.176 & $0.285 \pm 0.158$ & 0.934 & $0.391 \pm 0.194$ & 0.683 \\
\hline \multicolumn{8}{|l|}{ Follow-up visit } \\
\hline MetS present & 61 & $0.077 \pm 0.061$ & & $0.289 \pm 0.141$ & & $0.366 \pm 0.1622$ & \\
\hline Free of MetS & 60 & $0.112 \pm 0.091$ & 0.016 & $0.286 \pm 0.161$ & 0.930 & $0.399 \pm 0.197$ & 0.314 \\
\hline \multicolumn{8}{|l|}{ Individual MetS criteria } \\
\hline BP criterion present & 45 & $0.092 \pm 0.079$ & & $0.264 \pm 0.142$ & & $0.356 \pm 0.171$ & \\
\hline Free of BP criterion & 76 & $0.097 \pm 0.076$ & 0.627 & $0.304 \pm 0.151$ & 0.220 & $0.399 \pm 0.185$ & 0.206 \\
\hline FG criterion present & 53 & $0.078 \pm 0.067$ & & $0.290 \pm 0.132$ & & $0.369 \pm 0.162$ & \\
\hline Free of FG criterion & 68 & $0.107 \pm 0.085$ & 0.039 & $0.285 \pm 0.164$ & 0.846 & $0.394 \pm 0.194$ & 0.459 \\
\hline WC criterion present & 66 & $0.084 \pm 0.076$ & & $0.281 \pm 0.16$ & & $0.366 \pm 0.180$ & \\
\hline Free of WC criterion & 55 & $0.106 \pm 0.081$ & 0.124 & $0.295 \pm 0.13$ & 0.629 & $0.403 \pm 0.181$ & 0.266 \\
\hline HDL criterion present & 87 & $0.090 \pm 0.075$ & & $0.286 \pm 0.140$ & & $0.378 \pm 0.169$ & \\
\hline Free of HDL criterion & 34 & $0.105 \pm 0.088$ & 0.377 & $0.291 \pm 0.176$ & 0.876 & $0.396 \pm 0.208$ & 0.621 \\
\hline TG criterion present & 55 & $0.084 \pm 0.072$ & & $0.303 \pm 0.125$ & & $0.388 \pm 0.159$ & \\
\hline Free of TG criterion & 66 & $0.103 \pm 0.084$ & 0.063 & $0.274 \pm 0.169$ & 0.293 & $0.379 \pm 0.198$ & 0.78 \\
\hline Insulin resistance present & 49 & $0.072 \pm 0.068$ & & $0.286 \pm 0.142$ & & $0.365 \pm 0.167$ & \\
\hline No insulin resistance & 72 & $0.105 \pm 0.084$ & 0.055 & $0.288 \pm 0.157$ & 0.824 & $0.395 \pm 0.189$ & 0.506 \\
\hline \multicolumn{8}{|l|}{ Course of MetS } \\
\hline Mets at both visits & 46 & $0.063 \pm 0.054$ & & $0.276 \pm 0.126$ & & $0.339 \pm 0.146$ & \\
\hline Reverse MetS at follow-up & 9 & $0.115 \pm 0.088$ & 0.022 & $0.264 \pm 0.111$ & 0,795 & $0.380 \pm 0.175$ & 0.163 \\
\hline Free of MetS at both visits & 51 & $0.123 \pm 0.127$ & & $0.317 \pm 0.177$ & & $0.439 \pm 0.046$ & \\
\hline Newly developed MetS at follow-up & 16 & $0.111 \pm 0,092$ & 0.621 & $0.290 \pm 0.169$ & 0.611 & $0.403 \pm 0.028$ & 0.530 \\
\hline
\end{tabular}

Values are presented as mean \pm standard deviation.

MetS, metabolic syndrome; BP, blood pressure; FG, fasting glucose; WC, waist circumference; HDL, high density lipoprotein; TG, triglyceride. By Mann-Whitney $U$ test. 
Table 3. Relationship of serum bilirubin levels according to the number of MetS components

\begin{tabular}{|c|c|c|c|}
\hline Number of MetS criteria & Number (\%) & Direct bilirubin level (mg/dl) & Indirect bilirubin levels (mg/dl) \\
\hline \multicolumn{4}{|l|}{ Baseline } \\
\hline None & $10(7.6)$ & $0.140 \pm 0.078$ & $0.395 \pm 0.210$ \\
\hline Having 1 criteria for MetS & $31(23.7)$ & $0.113 \pm 0.087$ & $0.292 \pm 0.185$ \\
\hline Having 2 criteria for MetS & $29(22.1)$ & $0.117 \pm 0.099$ & $0.273 \pm 0.147$ \\
\hline Having 3 criteria for MetS & $29(22.1)$ & $0.089 \pm 0.065$ & $0.272 \pm 0.135$ \\
\hline Having 4 criteria for MetS & $24(18.3)$ & $0.072 \pm 0.082$ & $0.252 \pm 0.128$ \\
\hline Having 5 criteria for MetS & $8(6.1)$ & $0.075 \pm 0.052$ & $0.266 \pm 0.104$ \\
\hline \multicolumn{4}{|l|}{ Follow-up } \\
\hline None & $11(8.4)$ & $0.105 \pm 0.093$ & $0.267 \pm 0.148$ \\
\hline Having 1 criteria for MetS & $27(20.6)$ & $0.118 \pm 0.087$ & $0.307 \pm 0.187$ \\
\hline Having 2 criteria for MetS & $22(16.8)$ & $0.103 \pm 0.099$ & $0.270 \pm 0.133$ \\
\hline Having 3 criteria for MetS & $26(19.8)$ & $0.089 \pm 0.061$ & $0.293 \pm 0.149$ \\
\hline Having 4 criteria for MetS & $25(19.1)$ & $0.084 \pm 0.065$ & $0.304 \pm 0.143$ \\
\hline Having 5 criteria for MetS & $10(7.6)$ & $0.040 \pm 0.023$ & $0.240 \pm 0.110$ \\
\hline
\end{tabular}

Values are presented as number (\%) or mean \pm standard deviation.

MetS, metabolic syndrome.

MetS was significantly related to low serum direct bilirubin levels at the baseline, but this relationship was not evident at follow-up. Interestingly, at the follow-up visit, low serum direct bilirubin levels showed a significant relationship with the presence of fasting glucose level criteria and were associated with insulin resistance, nearly to a significance level, despite no association at baseline (Table 2).

The patients with MetS diagnosis and the patients who met the waist circumference criterion had lower total bilirubin levels compared to their counterparts. Indirect bilirubin levels showed only significant association with baseline waist circumference (Table 2). Serum total and indirect bilirubin did not show any significant association with the course of MetS.

Mean serum direct and indirect bilirubin levels of the patient based on the number of present MetS criteria are provided in Table 3. Linear regression analysis showed a significant relationship between direct bilirubin levels and the number of met criteria at baseline $(\mathrm{B}=-3,876$, adjusted $\mathrm{R}$ square $=0.048, p=0.007)$ and at follow-up evaluation $(\mathrm{B}=-3,734$, adjusted $\mathrm{R}$ square $=0.033, p=0.026)$. This relationship was not observed for indirect bilirubin levels $(p>0.05)$.

\section{Low versus High Bilirubin Groups}

These initial analyses revealed a consistent relationship between direct serum bilirubin levels and MetS, therefore we performed subsequent analyses based on a recent report on the lower risk of MetS in patients with serum bilirubin levels at upper percentiles. ${ }^{10)}$ We divided the patient into two groups: The patients within the first 50th percentile of serum direct bilirubin levels (low bilirubin group) and within the second 50th percentile (high bilirubin group).

MetS diagnosis was significantly more frequent in the low bilirubin group compared to the high bilirubin group at baseline (respectively $58.8 \%$ vs. $32.3 \%$ ) and follow-up (respectively $60.3 \%$ vs. $37.7 \%$ ) (Table 4). In the high bilirubin group, significantly more patients were free of the waist circumference criterion compared to the low bilirubin group at baseline (53.2\% vs. $26.1 \%)$ and follow-up $(56.6 \%$ vs. $36.8 \%)$. In this group, significantly more patients were also free of TG level criteria at baseline and fasting glucose level criteria at follow-up (Table 4). However, more patients were free of blood pressure criteria at baseline and TG level criteria at follow-up in the high bilirubin group, but the differences between groups did not prove significant. Low and high bilirubin groups were not different in respect to the presence of insulin resistance at baseline or follow-up.

Regarding the course of MetS at the end of six months, MetS diagnosis was still present in $90 \%$ of the patients with low bilirubin and $66.7 \%$ of patients in the high bilirubin group. The ratio of patients with reverse MetS was significantly higher in the high bilirubin group compared to the low bilirubin group (33.3\% vs. 10\%) (Table 4).

\section{Correlations between Bilirubin Levels and MetS Associated Parameters}

Consistent with the above findings, direct bilirubin levels showed a significant negative correlation with waist circumference $(\mathrm{r}=-0.178, p=0.042$ for both visits) and TG levels $(\mathrm{r}=-0.243, p=0.005$ for baseline; $\mathrm{r}=-0.185$, $p=0.042$ for follow-up). In addition, fasting blood glucose at follow-up showed a significant negative correlation 
Table 4. Comparison of MetS and its individual criteria in low and high bilirubin groups at baseline and follow-up visit

\begin{tabular}{|c|c|c|c|c|}
\hline Variable & $\begin{array}{l}\text { Low bilirubin group } \\
(0.01-0.09 \mathrm{mg} / \mathrm{dl})\end{array}$ & $\begin{array}{l}\text { High bilirubin group } \\
(0.10-0.30 \mathrm{mg} / \mathrm{dl})\end{array}$ & $\chi^{2}$ & $p$ value \\
\hline Female & $29(42.0)$ & $24(38.7)$ & 0.149 & 0.699 \\
\hline Male & $40(58.0)$ & $38(61.3)$ & & \\
\hline Baseline MetS present & $40(58.0)$ & $20(32.3)$ & 8.698 & 0.003 \\
\hline Free of MetS & $29(42.0)$ & $42(67.7)$ & & \\
\hline Follow-up MetS present & $41(60.3)$ & $20(37.7)$ & 6.063 & 0.014 \\
\hline Free of MetS & $27(39.7)$ & $33(62.3)$ & & \\
\hline \multicolumn{5}{|l|}{ Baseline individual MetS criteria } \\
\hline \multicolumn{5}{|l|}{ Blood pressure } \\
\hline Present $(n=35)$ & $20(44.1)$ & $15(28.3)$ & & \\
\hline Free of $(n=76)$ & $38(55.9)$ & $38(71.7)$ & 3.191 & 0.074 \\
\hline \multicolumn{5}{|l|}{ Fasting glucose } \\
\hline Present $(n=46)$ & $28(40.6)$ & $18(29.0)$ & 1.911 & 0.167 \\
\hline Free of $(n=85)$ & $41(59.4)$ & $44(71.0)$ & & \\
\hline \multicolumn{5}{|l|}{ Waist circumference } \\
\hline Present $(n=60)$ & $51(73.9)$ & $29(46.8)$ & 10.117 & 0.001 \\
\hline Free of $(n=51)$ & $18(26.1)$ & $33(53.2)$ & & \\
\hline \multicolumn{5}{|l|}{ HDL levels } \\
\hline Present $(\mathrm{n}=96)$ & $54(78.3)$ & $42(67.7)$ & 1.846 & 0.174 \\
\hline Free of $(n=35)$ & $15(21.7)$ & $20(32.3)$ & & \\
\hline \multicolumn{5}{|l|}{ Triglyceride levels } \\
\hline Present $(n=57)$ & $37(51.5)$ & $20(37.7)$ & 6.065 & 0.014 \\
\hline Free of $(n=74)$ & $32(48.5)$ & $42(62.3)$ & & \\
\hline Insulin resistance present $(n=33)$ & $21(30.4)$ & $12(19.4)$ & 2.127 & 0.145 \\
\hline No insulin resistance $(n=98)$ & $48(69.6)$ & $50(80.6)$ & & \\
\hline \multicolumn{5}{|l|}{ Follow-up individual MetS criteria } \\
\hline \multicolumn{5}{|l|}{ Blood pressure } \\
\hline Present $(n=45)$ & $30(44.1)$ & $15(28.3)$ & 3.190 & 0.074 \\
\hline Free of $(n=76)$ & $38(55.9)$ & $38(71.7)$ & & \\
\hline \multicolumn{5}{|l|}{ Fasting glucose } \\
\hline Present $(n=53)$ & $36(52.9)$ & $17(32.1)$ & 5.268 & 0.022 \\
\hline Free of $(n=68)$ & $32(47.1)$ & $36(67.9)$ & & \\
\hline \multicolumn{5}{|l|}{ Waist circumference } \\
\hline Present $(n=66)$ & $43(63.2)$ & $23(43.4)$ & 4.728 & 0.030 \\
\hline Free of $(n=55)$ & $25(36.8)$ & $30(56.6)$ & & \\
\hline \multicolumn{5}{|l|}{ HDL levels } \\
\hline Present $(n=87)$ & $51(75.0)$ & $36(67.9)$ & 0.738 & 0.390 \\
\hline Free of $(n=34)$ & $17(25.0)$ & $17(32.1)$ & & \\
\hline \multicolumn{5}{|l|}{ Triglyceride levels } \\
\hline Present $(n=55)$ & $35(51.5)$ & $20(37.7)$ & 2.266 & 0.093 \\
\hline Free of $(n=66)$ & $33(48.5)$ & $33(62.3)$ & & \\
\hline Insulin resistance present $(n=49)$ & $32(47.1)$ & $17(32.1)$ & 2.775 & 0.096 \\
\hline No insulin resistance $(n=72)$ & $36(52.9)$ & $36(67.9)$ & & \\
\hline \multicolumn{5}{|l|}{ Course of MetS } \\
\hline Continued MetS at both visits & $36(90.0)$ & $10(66.7)$ & 4.340 & 0.037 \\
\hline Reverse MetS at follow-up visit & $4(10.0)$ & $5(33.3)$ & & \\
\hline Free of Mets at both visits & $23(82.1)$ & $28(71.8)$ & 0.960 & 0.327 \\
\hline Newly developed MetS at follow-up visit & $5(17.9)$ & $10(28.2)$ & & \\
\hline
\end{tabular}

Values are presented as number (\%).

MetS, metabolic syndrome; HDL, high-density lipoprotein.

By Pearson chi-square analysis.

with direct bilirubin levels $(\mathrm{r}=-0,222, p=0.014)$.

Direct bilirubin levels did not show any significant correlations with weight, BMI, HOMA, or fasting insulin levels. No significant correlation was found between indirect bilirubin levels and MetS parameters. Serum bilirubin levels of smoking and non-smoking patients were not significantly different.

\section{DISCUSSION}

Our study has found that direct bilirubin levels are associated with the diagnosis and the course of MetS in patient 
with schizophrenia spectrum disorders. Our results are similar to those of previous studies conducted in general populations, overweight individuals, and patients with cardiovascular disorder. ${ }^{9-17,24-29)}$ In our study, the main MetS parameters associated with bilirubin levels were waist circumference and TG levels. Low serum direct bilirubin levels were found to be associated with baseline and follow-up MetS diagnosis, with the presence of waist circumference and TG level criteria at baseline, and with fasting glucose level criteria at follow-up. Serum indirect bilirubin levels only showed significant association with the presence of waist circumference criteria at baseline. Recently, it was demonstrated that bilirubin is a potent endogenous antioxidant and also plays a protective role in several stages of atherosclerotic inflammation. In cases of obesity and MetS where oxidative stress is increased, consumption of bilirubin increases, leading to a reduction in serum bilirubin levels which results in an increased risk of cardiovascular diseases by causing endothelial dysfunction. ${ }^{7,8,26,30)}$

\section{Relationship between Direct and Total Bilirubin and Metabolic Syndrome}

The relationship between serum direct bilirubin levels and MetS, as demonstrated in our study, has also been shown in many previous studies. ${ }^{8-13)}$ Jo et al. ${ }^{10)}$ have reported the bilirubin subtype that showed the most consistent relationship with MetS diagnosis as direct bilirubin in a sample of 5,321 patients. The relationship between direct bilirubin and MetS diagnosis observed in both visits in our study also supports this finding. We also observed that the more lower direct bilirubin levels were related to the more number of criteria for MetS were present as reported by Jenko-Pražnikar et al. ${ }^{26)}$ Recent studies suggest that the risk of MetS is increased two- to five-fold in patients with serum direct bilirubin levels that fall within the lower interquartile percentile (0th-75th percentile) as compared to patients within the uppermost percentile (75th-100th percentile) and an increase of 1 standard deviation in total bilirubin level reduces the risk of MetS by $17 \%$. Likewise, our study revealed that MetS diagnosis was significantly lower in the high bilirubin group. In these patients, reversed MetS rate was also found to be significantly higher than the low bilirubin group. In conclusion, these findings, consistent with the literature, suggest that high direct serum bilirubin levels may be associated with a lower risk of MetS in schizophrenia patients undergoing antipsychotic treatment.

\section{Relationship between Serum Bilirubin and MetS Parameters}

\section{Abdominal obesity}

While waist circumference was significantly associated with all bilirubin forms at baseline, it was associated with only direct bilirubin levels at follow-up. Direct bilirubin levels were significantly lower in patients who met the MetS criterion for waist circumference at baseline and follow-up evaluation as compared to patients who did not meet this criterion. In addition, the presence of waist circumference criteria was significantly higher in the low bilirubin group as compared to the high bilirubin group at the baseline and follow-up visits. Correlation analyses also showed a significant negative correlation between waist circumference and direct bilirubin levels. Consistent with our results, the literature reports that abdominal obesity alone has been correlated with low serum bilirubin levels. ${ }^{8,11-13,24,25)}$ Choi et al. ${ }^{8)}$ have reported an inverse relationship between the high levels of bilirubin and abdominal obesity. Serum bilirubin was found to be inversely associated with the amount of visceral adipose tissue in 2,450 subjects examined in a Swedish Obese Subjects trial and this finding was considered a reflection of MetS in obese individuals. ${ }^{31)}$ Kwon et al. ${ }^{13)}$ have examined the bilirubin levels in women by dividing them into four categories by interquartile ranges and reported a significant relationship between high levels of bilirubin and lower waist circumference. Jenko-Pražnikar et al. $^{26)}$ have also reported serum bilirubin levels were negatively associated with abdominal obesity in overweight asymptomatic middle-aged individuals. Distinct from above mentioned studies, Andersson et al. ${ }^{32)}$ have reported in the results of their study that weight loss causes an increase in serum bilirubin concentration. Abovementioned studies and our study have pointed out the relationship between serum bilirubin levels and obesity.

\section{Dyslipidemia and abnormal glucose metabolism}

In our study, TG levels were associated with lower direct bilirubin concentrations. However, baseline and follow-up data were partially inconcistent. Correlation analyses showed that the negative relationship was maintained between direct bilirubin and TG levels at both baseline and follow-up. However, direct bilirubin levels were significantly lower in patients who met the MetS criterion for TG levels compared to patients who didn't meet this criterion at baseline; the differences between these two groups was not present at follow-up. Similarly, the presence of the 
MetS criterion for TG levels at baseline was significantly lower in the high bilirubin group, but this relationship was less apperent at follow-up. The relationship between bilirubin and lipid profile was first reported by Breimer et al. ${ }^{28)}$ in a large cohort of middle-aged, British men. This study indicated lower concentrations of TG and higher concentrations of HDL in individuals with higher bilirubin concentrations. A number of subsequent studies described negative relationships between bilirubin and TG levels $^{8,24,26,29)}$ as well as bilirubin showed a positive association with HDL concentrations. ${ }^{8,13,24,27,30)}$ Chang et al. ${ }^{24)}$ reported that serum direct bilirubin levels were inversely associated with total cholesterol, low-density lipoprotein (LDL) cholesterol, and TG, and positively associated with HDL cholesterol levels. Yoshino et al. ${ }^{30)}$ reported a positive correlation between total bilirubin and HDL levels in an overweight group. Onat $e a{ }^{27)}$ reported a significant linear relationship between bilirubin levels and HDL cholesterol in men. Jenko-Pražnikar et al. ${ }^{26)}$ also reported that serum bilirubin levels were negatively associated with FTG, total cholesterol, and LDL cholesterol in overweight individuals.

In our study, lipids other than TG showed no relationship with bilirubin levels, which may be due to several reasons. Most of the previously published studies were conducted with large sample sizes, whereas the relatively small sample size in this study may limit the validity of our results. Additionally, atypical antipsychotics have been shown to have negative effects on lipid profile (levels of TG, total cholesterol, HDL cholesterol, etc.). ${ }^{6,33)}$ Most of our patients were treated with atypical antipsychotics, which may have acted as a confounding factor on our results. Some studies also suggest that the relationship between bilirubin levels and serum lipids may vary based on sex. ${ }^{26,27)}$ Further well-controlled studies on the relationship between serum lipid profile and bilirubin levels in patients undergoing antipsychotic treatment, controlling for the effect of confounding factors, may provide more information about this issue. Nevertheless, our findings are consistent with the literature in that there is a relationship between low levels of bilirubin and high levels of triacylglycerol, which is the most remarkable result of most studies examining this matter in healthy subjects. ${ }^{34)}$

In our study, serum direct bilirubin levels were significantly lower in patients who met the MetS criterion for fasting glucose levels only at follow-up visit as compared to patients who were free of this criterion. At follow-up, low serum direct bilirubin levels tended to accompany the presence of insulin resistance. There were no relationships observed between the bilirubin levels and fasting insulin levels or HOMA-IR values. Although our results suggest a limited relationship between direct bilirubin levels and glucose metabolism in patients using antipsychotics, the results do not allow for a conclusion to be made on this matter. Previous studies have reported that serum total bilirubin concentrations were inversely associated with hyperinsulinemia, insulin resistance, and systemic inflammation. ${ }^{12,26)}$ The absence of a consistent relationship between bilirubin levels and glucose metabolism in our study, distinct from previous studies, may be attributed to characteristics of our patient group, such as the limited sample size and use of antipsychotics. A study conducted in a general population with 1,052 Turkish adult patients showed that total bilirubin levels are associated with insulin resistance but not associated with abdominal obesity. ${ }^{27)}$ This, as in our study, suggests that the relationship of bilirubin levels with MetS and its parameters in patients using antipsychotics, may have different characteristics than those observed in the general population. However, more studies are required on this issue.

Oxidative stress is believed to play a pathophysiological role in schizophrenia. Studies addressing the relationship between bilirubin levels and schizophrenia or psychosis report conflicting results. ${ }^{35,36)}$ Studies also suggest that oxidative stress is involved in the pathogenesis of MetS and as a natural antioxidant, bilirubin may play a protective role against oxidative stress and atherosclerotic processes in healthy individuals. ${ }^{7,8,30)}$ Our results suggest that bilirubin may also involve in MetS accompanied to schizophrenia. However, as our research is the first study on this issue, these conclusions must be supported with future studies.

The most important result of this study may be summarized as follows: Firstly, direct bilirubin levels are associated with several MetS associated parameters, including MetS diagnosis, abdominal obesity, and TG levels. Secondly, high levels of direct serum bilirubin were associated with a lower risk of MetS in patients with schizophrenia spectrum disorders using antipsychotics. Our study is the first to investigate the association of bilirubin with MetS and its parameters in patients with schizophrenia spectrum disorders. The key strength of our study is that it covers the longitudinal effects of baseline serum bilirubin concentrations on incident MetS for six-month follow-up period. The relationships of MetS diagnosis and abdominal obesity with direct bilirubin levels remained stable during the six-month follow-up period. This suggests that the direct bilirubin value measured at any given 
time may be a useful predictor for abdominal obesity. Jenko-Pražnikar et al. ${ }^{26)}$ also reported that a low level of bilirubin in non-symptomatic individuals may be an early biomarker of MetS. We could not find a study that prospectively investigates the stability of the relationship between bilirubin levels and MetS parameters in the literature. Most of the atypical antipsychotics currently used in the treatment of schizophrenia increase the risk of MetS and worsen metabolic parameters, beginning soon after treatment initiation. ${ }^{6,33)}$ A factor with a stable relationship with MetS over time, may be used as a predictor to reduce the risk of MetS. High levels of direct bilirubin in patients with schizophrenia spectrum disorders may be a cost-effective indicator to demonstrate lower risk of MetS. In addition, antipsychotics that have strong metabolic side effects may be avoided in patients with low serum direct bilirubin levels, as to reduce the interference when evaluating for the risk of MetS. However long-term studies with a larger sample are required on this matter. Important limitations of our study include our limited sample size and the absence of a control group. Another limitation of our study is the widespread use of antipsychotics in our patient population. Although it was reported that antipsychotics do not affect the levels of serum bilirubin, ${ }^{19)}$ we can not exclude the possible confounding effect of antipsychotics. An investigation on the effects of antipsychotics on MetS and bilirubin levels starting from the initiation of therapy would better clarify this matter.

Our study suggests that the limited, yet stable relationship of bilirubin with abdominal obesity, the key characteristic of MetS, may be worth further investigating.

\section{REFERENCES}

1. Alberti KG, Zimmet P, Shaw J. Metabolic syndrome--a new world-wide definition. A Consensus Statement from the International Diabetes Federation. Diabet Med 2006;23: 469-480.

2. Saha S, Chant D, McGrath J. A systematic review of mortality in schizophrenia: is the differential mortality gap worsening over time? Arch Gen Psychiatry 2007;64:11231131.

3. Druss BG, Rohrbaugh RM, Levinson CM, Rosenheck RA. Integrated medical care for patients with serious psychiatric illness: a randomized trial. Arch Gen Psychiatry 2001;58: 861-868.

4. Newcomer JW. Second-generation (atypical) antipsychotics and metabolic effects: a comprehensive literature review. CNS Drugs 2005;19 Suppl 1:1-93.

5. Henderson DC, Vincenzi B, Andrea NV, Ulloa M, Copeland PM. Pathophysiological mechanisms of increased cardiometabolic risk in people with schizophrenia and other severe mental illnesses. Lancet Psychiatry 2015;2:452-464.

6. Hasnain M, Fredrickson SK, Vieweg WV, Pandurangi AK. Metabolic syndrome associated with schizophrenia and atypical antipsychotics. Curr Diab Rep 2010;10:209-216.

7. Vitek L. The role of bilirubin in diabetes, metabolic syndrome, and cardiovascular diseases. Front Pharmacol 2012;3: 55.

8. Choi SH, Yun KE, Choi HJ. Relationships between serum total bilirubin levels and metabolic syndrome in Korean adults. Nutr Metab Cardiovasc Dis 2013;23:31-37.

9. Hwang HJ, Kim SH. Inverse relationship between fasting direct bilirubin and metabolic syndrome in Korean adults. Clin Chim Acta 2010;411:1496-1501.

10. Jo J, Yun JE, Lee H, Kimm H, Jee SH. Total, direct, and indirect serum bilirubin concentrations and metabolic syndrome among the Korean population. Endocrine 2011; 39:182-189.

11. Lin LY, Kuo HK, Hwang JJ, Lai LP, Chiang FT, Tseng CD, et al. Serum bilirubin is inversely associated with insulin resistance and metabolic syndrome among children and adolescents. Atherosclerosis 2009;203:563-568.

12. Wu Y, Li M, Xu M, Bi Y, Li X, Chen Y, et al. Low serum total bilirubin concentrations are associated with increased prevalence of metabolic syndrome in Chinese. J Diabetes 2011;3:217-224.

13. Kwon KM, Kam JH, Kim MY, Kim MY, Chung CH, Kim $\mathrm{JK}$, et al. Inverse association between total bilirubin and metabolic syndrome in rural Korean women. $J$ Womens Health (Larchmt) 2011;20:963-969.

14. Erdogan D, Gullu H, Yildirim E, Tok D, Kirbas I, Ciftci $\mathrm{O}$, et al. Low serum bilirubin levels are independently and inversely related to impaired flow-mediated vasodilation and increased carotid intima-media thickness in both men and women. Atherosclerosis 2006; 184:431-437.

15. Perlstein TS, Pande RL, Beckman JA, Creager MA. Serum total bilirubin level and prevalent lower-extremity peripheral arterial disease: National Health and Nutrition Examination Survey (NHANES) 1999 to 2004. Arterioscler Thromb Vasc Biol 2008;28:166-172.

16. Ko GT, Chan JC, Woo J, Lau E, Yeung VT, Chow CC, et al. Serum bilirubin and cardiovascular risk factors in a Chinese population. J Cardiovasc Risk 1996;3:459-463.

17. Novotný L, Vítek L. Inverse relationship between serum bilirubin and atherosclerosis in men: a meta-analysis of published studies. Exp Biol Med (Maywood) 2003;228: 568-571.

18. Vítek L, Jirsa M, Brodanová M, Kalab M, Marecek Z, Danzig V, et al. Gilbert syndrome and ischemic heart disease: a protective effect of elevated bilirubin levels. Atherosclerosis 2002;160:449-456.

19. Atasoy N, Erdogan A, Yalug I, Ozturk U, Konuk N, Atik $\mathrm{L}$, et al. A review of liver function tests during treatment with atypical antipsychotic drugs: a chart review study. Prog Neuropsychopharmacol Biol Psychiatry 2007;31: 1255-1260.

20. Balci Sengul C, Kalkanci O, Karadag F, Sengul C. Antipsychotics and metabolic syndrome: a naturalistic six-month follow-up study. Bull Clin Psychopharmacol 2013;23(Suppl 1): $\$ 138$.

21. Andreasen NC. Scale for the assessment of negative symptoms (SANS). Iowa City:Department of Psychiatry College of Medicine, The University of Iowa;1983.

22. Andreasen NC. Scale for the assessment of positive symptoms (SAPS). Iowa City:Department of Psychiatry College of Medicine, The University of Iowa;1984.

23. Physical status: the use and interpretation of anthropometry. Report of a WHO Expert Committee. World Health Organ Tech Rep Ser 1995;854:1-452. 
24. Chang Y, Ryu S, Zhang Y, Son HJ, Kim JY, Cho J, et al. A cohort study of serum bilirubin levels and incident non-alcoholic fatty liver disease in middle aged Korean workers. PLoS One 2012;7:e37241.

25. Giral P, Ratziu V, Couvert P, Carrié A, Kontush A, Girerd $\mathrm{X}$, et al. Plasma bilirubin and gamma-glutamyltransferase activity are inversely related in dyslipidemic patients with metabolic syndrome: relevance to oxidative stress. Atherosclerosis 2010;210:607-613.

26. Jenko-Pražnikar Z, Petelin A, Jurdana M, Žiberna L. Serum bilirubin levels are lower in overweight asymptomatic middle-aged adults: an early indicator of metabolic syndrome? Metabolism 2013;62:976-985.

27. Onat A, Ozhan H, Karabulut A, Albayrak S, Can G, Hergenc G. Serum bilirubin levels in Turkish adults show inverse relation with insulin resistance and overall obesity, without association with metabolic syndrome. Turk Kardiyol Dern Ars 2007;35:28-36.

28. Breimer LH, Wannamethee G, Ebrahim S, Shaper AG. Serum bilirubin and risk of ischemic heart disease in middle-aged British men. Clin Chem 1995;41:1504-1508.

29. McArdle PF, Whitcomb BW, Tanner K, Mitchell BD, Shuldiner AR, Parsa A. Association between bilirubin and cardiovascular disease risk factors: using Mendelian randomization to assess causal inference. BMC Cardiovasc Disord 2012;12:16.

30. Yoshino S, Hamasaki S, Ishida S, Kataoka T, Yoshikawa A, Oketani N, et al. Relationship between bilirubin concen- tration, coronary endothelial function, and inflammatory stress in overweight patients. $J$ Atheroscler Thromb 2011;18:403-412.

31. Torgerson JS, Lindroos AK, Sjöström CD, Olsson R, Lissner L, Sjöström L. Are elevated aminotransferases and decreased bilirubin additional characteristics of the metabolic syndrome? Obes Res 1997;5:105-114.

32. Andersson C, Weeke P, Fosbøl EL, Brendorp B, Køber L, Coutinho W, et al; SCOUT Executive Steering Committee; SCOUT investigators. Acute effect of weight loss on levels of total bilirubin in obese, cardiovascular high-risk patients: an analysis from the lead-in period of the Sibutramine Cardiovascular Outcome trial. Metabolism 2009;58:11091115.

33. Cohn T, Prud'homme D, Streiner D, Kameh H, Remington G. Characterizing coronary heart disease risk in chronic schizophrenia: high prevalence of the metabolic syndrome. Can J Psychiatry 2004;49:753-760.

34. Bulmer AC, Verkade HJ, Wagner KH. Bilirubin and beyond: a review of lipid status in Gilbert's syndrome and its relevance to cardiovascular disease protection. Prog Lipid Res 2013;52:193-205.

35. Bach DR, Kindler J, Strik WK. Elevated bilirubin in acute and transient psychotic disorder. Pharmacopsychiatry 2010;43:12-16.

36. Radhakrishnan R, Kanigere M, Menon J, Calvin S, Janish A, Srinivasan K. Association between unconjugated bilirubin and schizophrenia. Psychiatry Res 2011;189:480-482. 